Original Paper http://ajol.info/index.php/ijbcs http://indexmedicus.afro.who.int

\title{
Importance de la "Teigne du chou" dans les Niayes au Sénégal : interrelations avec la température et les cultivars utilisés
}

\author{
Babacar LABOU ${ }^{1 *}$, Dominique BORDAT ${ }^{1}$, Thierry BREVAULT ${ }^{2,3}$ et \\ Karamoko DIARRA ${ }^{1}$ \\ ${ }^{1}$ UCAD, Equipe Production et Protection Intégrées en Agroécosystèmes Horticoles - 2PIA, \\ Faculté des Sciences et Techniques, Dakar, Sénégal. \\ ${ }^{2}$ BIOPASS, ISRA-UCAD-IRD, Dakar, Sénégal. \\ ${ }^{3}$ CIRAD, UPR AIDA, F-34398 Montpellier, France. \\ *Auteur correspondant. E-mail: babslabou@yahoo.fr; Tel : +221-352-63-30
}

\section{REMERCIEMENTS}

Ce travail a été soutenu par l'IRD (AIRD) sous Grant PAIRS-BIOBIO 2013.

\section{RESUME}

L'importance des populations de Plutella xylostella dans la zone des Niayes est étudiée en relation avec la température et les cultivars utilisés par les agriculteurs. Pour cela, trois zones sont choisies pour un nombre total de 59 parcelles réparties comme suit ; 20 au sud, 22 au centre et 17 au nord. Elles sont échantillonnées tous les 21 jours selon la zone et 24 plants dans chaque parcelle sont observés. Les échantillonnages sont effectués sur deux saisons; d'octobre à janvier et de février à mai. Les données climatiques sont recueillies à l'aide d'enregistreurs de température et d'humidité relative installés dans six parcelles (deux par zone). Chaque parcelle est numérotée et géo-référencée par GPS. Le nombre d'individu de P. xylostella est plus important respectivement au Sud, au Centre et au Nord. Une corrélation est établie entre le nombre d'individus du ravageur et la température. Les différents cultivars utilisés par les agriculteurs, dans les trois zones (Sud, Centre et Nord), ont une réponse similaire par rapport aux attaques des populations de la teigne. Leur tolérance diminue quand la pression parasitaire augmente.

(C) 2016 International Formulae Group. All rights reserved.

Mots clés : Plutella xylostella, Brassicacées, Afrique de l'Ouest, dégâts, donnée climatique, spéculation.

\section{Importance of the diamondback moth in the Niayes in Senegal: relations with temperature and cabbage cultivars}

\section{ABSTRACT}

Importance of populations of diamondback moth in the Niayes area is studied in relation to temperature and cabbage cultivars used by farmers. Three areas were chosen for a total of 59 cabbage fields as follows: 20 in South, 22 in Center and 17 in the North of Niayes. Plots were sampled every 21 days and 24 plants in each plot were observed. Sampling was carried over two seasons: from October to January and from February to May. Climate data were collected using temperature and relative humidity recorders installed in six plots (two 
per zone). Each plot was numbered and georeferenced by GPS. The number of P. xylostella was greater in the South than in the Centre and North. A correlation was observed between abundance of the pest and temperature. Infestation of the diamondback moth populations were not influenced by cultivar. Their tolerance decreased as pest pressure increased.

(C) 2016 International Formulae Group. All rights reserved.

Keywords: Plutella xylostella, Brassicaceae, West Africa, damage, climate data.

\section{INTRODUCTION}

Les Brassicacées représentent l'une des plus importantes ressources alimentaires mondiales (Talekar and Shelton, 1993). Cette famille comprend 350 genres dont 3500 espèces cultivées et sauvages (Warwick et al., 2003). Les choux constituent une importante source alimentaire et de revenus pour les populations rurales et urbaines en termes de production, de commercialisation et de transformation (Grzywacz et al., 2010). D'après la FAO (FAOSTAT, 2012), ils contribuent aujourd'hui à plus de 26 milliards \$ US dans l'économie mondiale. En Afrique de l'Ouest, plus particulièrement au Sénégal, les choux sont cultivés sur 3500 hectares avec une production annuelle estimée à 60.000 tonnes (FAO, 2015).

Actuellement, au Sénégal, le chou est cultivé pendant toute l'année et il est couramment utilisé dans différents plats nationaux comme le riz au poisson (Thiébou djène), la sauce d'arachide... De ce fait, il constitue l'une des principales spéculations maraîchères dans la zone des Niayes.

Malheureusement, cette culture est fréquemment soumise aux attaques de plusieurs insectes ravageurs parmi lesquels Plutella xylostella (L.) lépidoptère défoliateur (teigne des brassicacées). Cette espèce provoque des pertes estimées à plus de $90 \%$ de la production totale (Shelton, 2004 ; Sarfraz et al., 2005). Au Sénégal, ce taux est estimé entre $51 \%$ et $94 \%$.

L'emploi des insecticides de synthèse constitue la principale méthode de lutte dans le monde contre ce ravageur (Furlong et al., 2012). Malheureusement, ces pesticides entraînent plusieurs problèmes:(i) l'élimination des ennemis naturels (Furlong et al., 2012); (ii) l'augmentation du coût de production (Chilcutt and Tabashnik, 1997) ; (iii) l'apparition de souches résistantes (Hooks and Johnson, 2003 ; Macharia et al., 2005 ; Sarfraz and Keddie, 2005 ; Shelton et al., 2007 ; Huang et al., 2010); (iv) l'intoxication des populations (Badiane et al., 2015). De ce fait, d'autres méthodes de lutte se développent, privilégiant une gestion durable des populations d'insectes. Aussi, elles évitent des dégâts économiques et préservent l'environnement et la santé humaine (Hill and Foster, 2000 ; Sow, 2013).

Les objectifs de cette étude sont: (i) de connaître la distribution des populations de la teigne dans les cultures de choux des Niayes ; (ii) d'identifier l'importance des dégâts de $P$. xylostella sur les cultures ; (iii) de proposer des stratégies de gestion durable des populations contre ce ravageur.

\section{MATERIEL ET METHODES \\ Sites d'échantillonnages}

Avec près de $80 \%$ de la production nationale, la zone des Niayes est le levier incontestable du développement horticole du Sénégal (Figure 1). Cette zone s'étend sur une longueur de $180 \mathrm{~km}$ bordant la frange maritime du nord du pays partant de Dakar à Saint-Louis en passant par la bordure ouest de Thiès et de Louga. Sa largeur varie de cinq à $30 \mathrm{~km}$ à l'intérieur des terres.

Elle présente des caractéristiques pédologiques, hydrologiques et climatiques favorables à la production horticole, en particulier le marâichage et l'arboriculture fruitière. Elle est constituée de dépressions inter dunaires présentant des sols riches en matière organique où la nappe phréatique est affleurante et des sols ferrugineux non lessivés moins riches, où l'eau est accessible au moyen de forages. 
$\mathrm{Au}$ cours de cette étude, le choix a porté sur trois zones le long d'un transect Dakar-Saint-Louis. Les échantillonnages ont été effectués sur deux saisons : (i) la première d'octobre à janvier et la seconde de février à mai.

\section{Echantillonnage}

Pour la première saison, 32 parcelles de choux ont été sélectionnées sur les trois zones des Niayes, (10 parcelles au Sud, 12 au Centre et 10 au Nord). Chaque parcelle choisie est distante d'au moins deux $\mathrm{km}$ de l'autre. Les dates de repiquage sont étalées sur plus de deux mois et demi. Pour la seconde saison, 27 parcelles (10 dans la zone Sud, 10 au Centre et sept au Nord) ont été échantillonnées. Chaque parcelle est numérotée et géoréférencée par GPS (Tableau 1).

Lors de nos échantillonnages, nous avons été confrontés à des plants de chou n'étant pas au même stade phénologique entre quelques parcelles. En effet, cette hétérogénéité est due aux agriculteurs qui ne repiquent pas leurs choux à la même période (étalement sur plus de deux mois). L'inégalité du nombre de parcelles échantillonnées dans les trois zones est également due aux producteurs, par exemple à cette période (février - mai), dans la zone nord, la culture de l'oignon est préférée à celle du chou.

Pour la mesure des facteurs climatiques, des enregistreurs de température
(Hobo, Prosensor) à 2 minutes d'intervalle de prise, sont installés dans six parcelles (deux par zone).

Le suivi par zone des parcelles se fait une fois tous les 21 jours, depuis le repiquage jusqu'à la première date de récolte et cela pour chaque saison. Pendant la première saison, nous avons effectué six échantillonnages dans chaque zone et cinq échantillonnages pendant la deuxième saison.

Dans chaque parcelle, 24 plants sont observés de façon aléatoire en comptant le nombre de chenilles et de nymphes présentes sur chaque plant identifié.

Une enquête parallèles sur les pratiques culturales y compris les cultivars et les pesticides utilisés par les agriculteurs, a été réalisée afin d'évaluer leur influence sur les infestations selon la zone.

\section{Analyses statistiques}

L'évolution des populations de chenilles de $P$. xylostella en relation avec la température a été analysée en utilisant la corrélation de Pearson coefficient (r). Des modèles d'effets mixtes linéaires ont été utilisés pour examiner les effets de zone (Sud, Centre, Nord de Niayes) et le temps de la saison (début vs fin de saison) sur la température. Les calculs ont été effectués en utilisant le logiciel XLstat (version 1.1.2012).

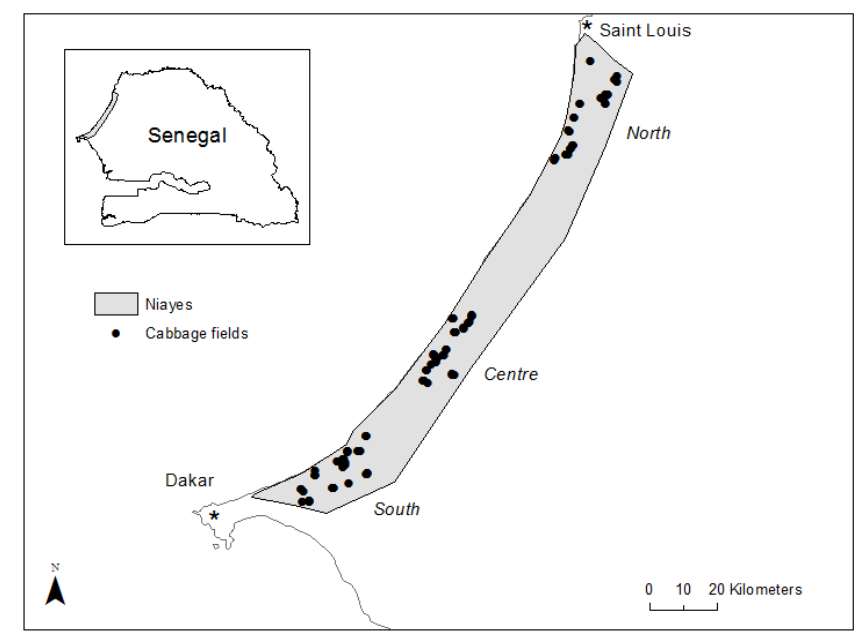

Figure 1 : Carte des sites d'échantillonnages des zones Sud, Centre et Nord. 


\section{RESULTATS}

Importance des populations de Plutella xylostella échantillonnées dans les zones sud, centre et nord

Nous constatons une répartition inégale du nombre moyen d'individus (chenilles et nymphes) de $P$. xylostella suivant les trois zones. La zone sud est la plus infestée suivie du centre et enfin le nord. Au sud, deux parcelles sur 20 ne présentent pas de chenille. Une en totalise plus de cinq, les 17 dernières comportent d'une à quatre chenilles par chou. Au centre, huit parcelles n'ont pas de chenille, deux en possèdent cinq, les 12 dernières totalisent entre une à trois chenilles par chou. $\mathrm{Au}$ nord, huit parcelles sur 17 n'ont aucune chenille, parmi les neuf parcelles restantes seule une parcelle a plus de deux chenilles par chou (Figure 2).

\section{Echantillonnage par zones}

Dans la zone sud, nous observons un maximum de 1235 individus dans la parcelle 13, (ce qui fait une moyenne de 13 individus/chou), contre un minimum de sept dans la parcelle deux (0 individu/chou). Six parcelles ont chacune un total de moins de 100 individus (un maximum d'un/chou), sept entre 100 et 200 (soit en moyenne deux/chou), cinq entre 200 et 300 (soit trois individus/chou). Deux parcelles présentent chacune un nombre d'individus supérieurs à 300 (quatre individus/chou).

Six cultivars de chou ont été utilisés par les agriculteurs dans cette zone (Tableau 2).

La zone centre présente un maximum de 377 individus au niveau de la parcelle 122 (soit cinq individus/chou) et un pour la 27 (zéro/chou). Treize parcelles sur 22 en ont chacune moins de 100 (un/chou), six parcelles entre 100 et 200 (deux/chou) et deux en ont plus de 300 (cinq/chou).

Cinq cultivars différents sont utilisés par les agriculteurs (Tableau 3).

Le maximum d'individus de la zone nord est de 208 (trois/chou) au niveau de la parcelle 148, le minimum est d'un individu pour la 55 (0/chou). Douze parcelles sur 17 présentent chacune moins de 100 individus (un/chou) tandis que quatre en ont entre 100 et 200 (deux/chou).

Six cultivars différents sont utilisés par les agriculteurs (Tableau 4).

\section{Evolution des populations de $P$. xylostella par rapport à la température}

Dans la zone sud, on note une diminution progressive de la température à partir du début des échantillonnages le 9 octobre $\left(29,2{ }^{\circ} \mathrm{C}\right)$ jusqu'au 5 mars $\left(20,5{ }^{\circ} \mathrm{C}\right)$. Ensuite la température augmente sensiblement jusqu'au 7 mai $\left(22,4{ }^{\circ} \mathrm{C}\right)$. Dans le même temps, la population de $P$. xylostella varie de manière irrégulière, 18 individus le 9 octobre, 85 le 31 octobre, 55 le 12 décembre, 123 le 21 janvier et 65 récoltés le 16 avril. Il n'y a aucune corrélation $(r=0,64 ; P=0,12)$ entre les courbes de température et de population de P. xylostella (Figure 2).

Dans la zone centre, on note une diminution progressive et régulière de la température, $29^{\circ} \mathrm{C}$ le 17 octobre au début des échantillonnages jusqu'à $20,6{ }^{\circ} \mathrm{C}$ le 23 avril. La température remonte après cette date, 22,8 ${ }^{\circ} \mathrm{C}$ le 14 mai, date du dernier échantillonnage.

En parallèle, les populations du ravageur, absentes au début des échantillonnages le 17 octobre, augmentent fortement jusqu'au 29 janvier avec 282 individus récoltés, puis chutent rapidement jusqu'au 14 mai, seulement 30 individus. Comme dans la zone sud, il n'y a aucune corrélation $(\mathrm{r}=0,62 ; \mathrm{P}=0,36)$ entre la température et les populations de $P$. xylostella (Figure 3).

Dans la zone nord, la température diminue régulièrement, $29,7{ }^{\circ} \mathrm{C}$ le 23 octobre lors du début des échantillonnages, $22,1^{\circ} \mathrm{C}$ le 26 décembre. Puis un plateau apparaît jusqu'au 30 avril avec une faible fluctuation des températures, de 21,9 à $22,8^{\circ} \mathrm{C}$. Celle-ci remonte pour le dernier échantillon, $24,8^{\circ} \mathrm{C}$ le 21 mai. Durant la première saison, il y a très peu d'individus, le maximum récolté étant de 30. Au cours de la seconde, les populations augmentent régulièrement de façon significative, de trois à 72 . Elles sont significativement corrélées $(r=0,882$; 
$\mathrm{P}<0,05)$ avec l'augmentation de la température alors qu'elles ne le sont pas en première saison (Figure 4).

Sensibilité des cultivars envers les populations de la Teigne

Il ne semble pas y avoir de différence de sensibilité entre les cultivars utilisés par les producteurs vis à vis des populations de $P$. xylostella. En effet, le nombre des individus de la teigne qui sont récoltés sur les choux varient peu; de 1,89 à 3,80 respectivement pour Tropicana et Tropica Cross dans la zone sud, de 0,24 à 1,44 respectivement pour Tropica Leader et Dragon dans le centre et de 0,16 à 1,71 pour K K Cross et Taizé (Tableau $5)$.

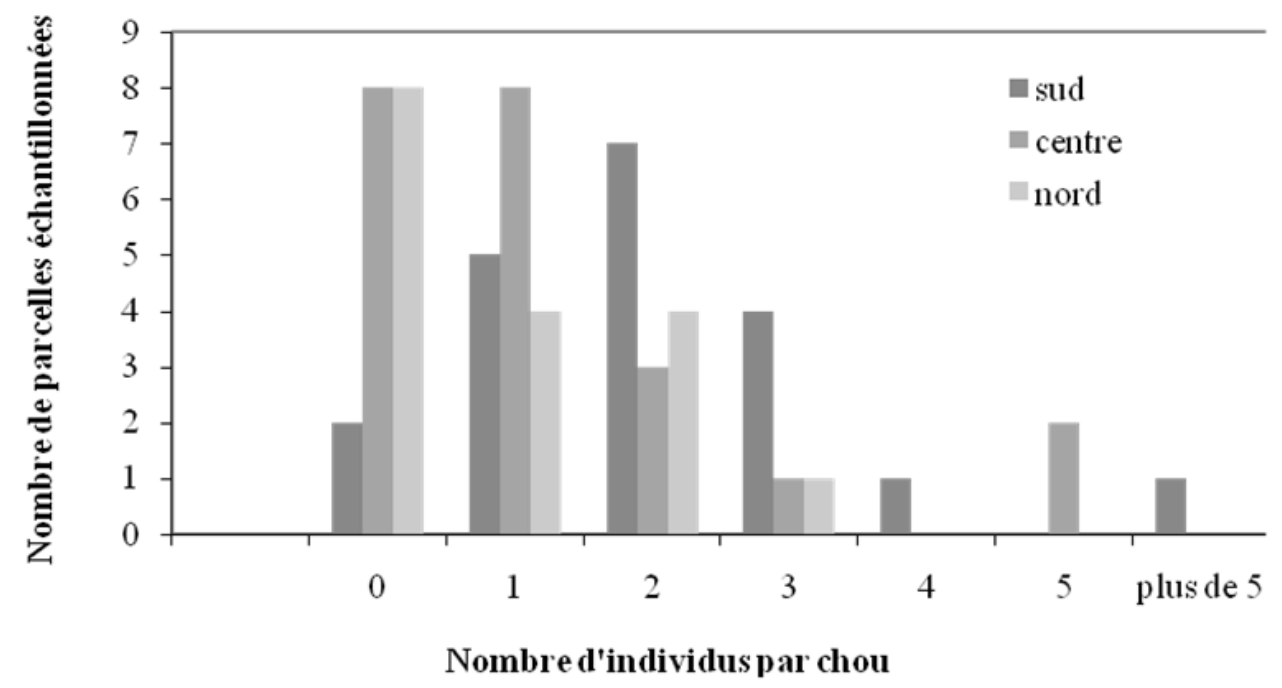

Figure 1 : Nombre moyen d'individus (chenilles et nymphes) de $P$. xylostella présents sur les choux dans les zones sud, centre et nord des Niayes.

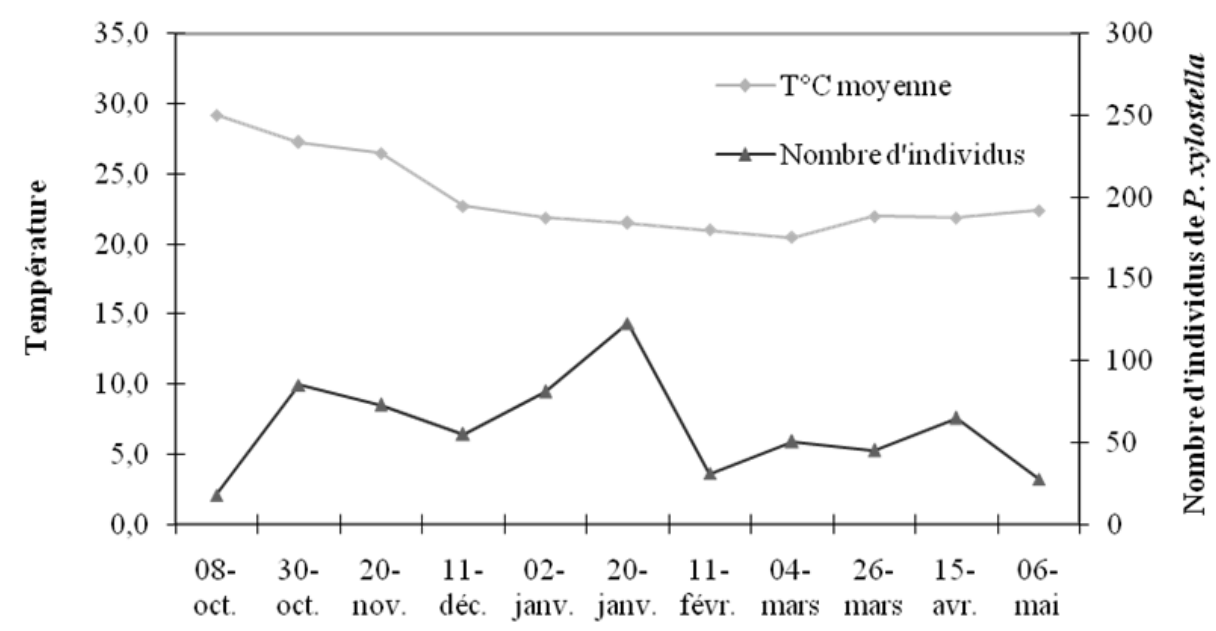

Date des échantillonnages

Figure 2: Evolution du nombre de P. xylostella (chenilles et nymphes) suivant la température dans la zone Sud. 


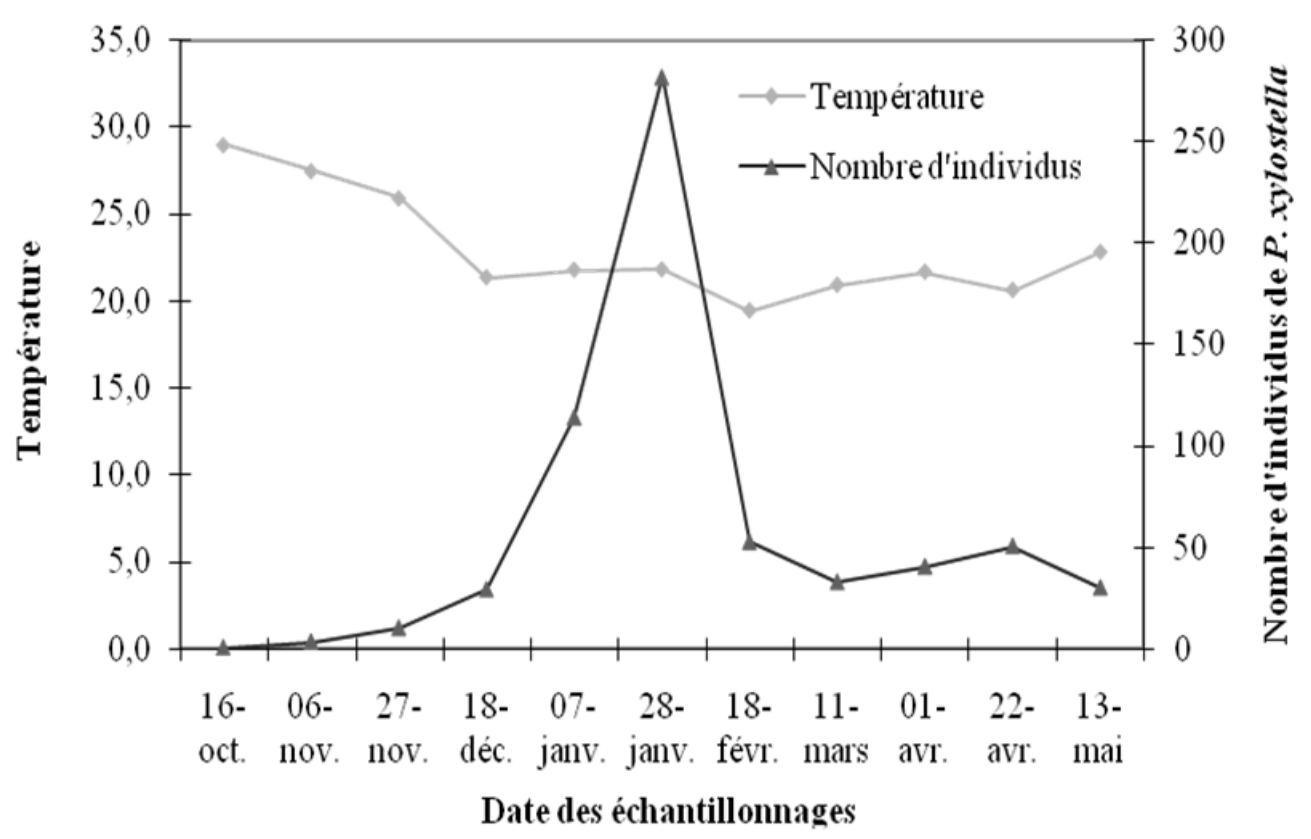

Figure 3: Evolution du nombre de P. xylostella (chenilles et nymphes) suivant la température dans la zone Centre.

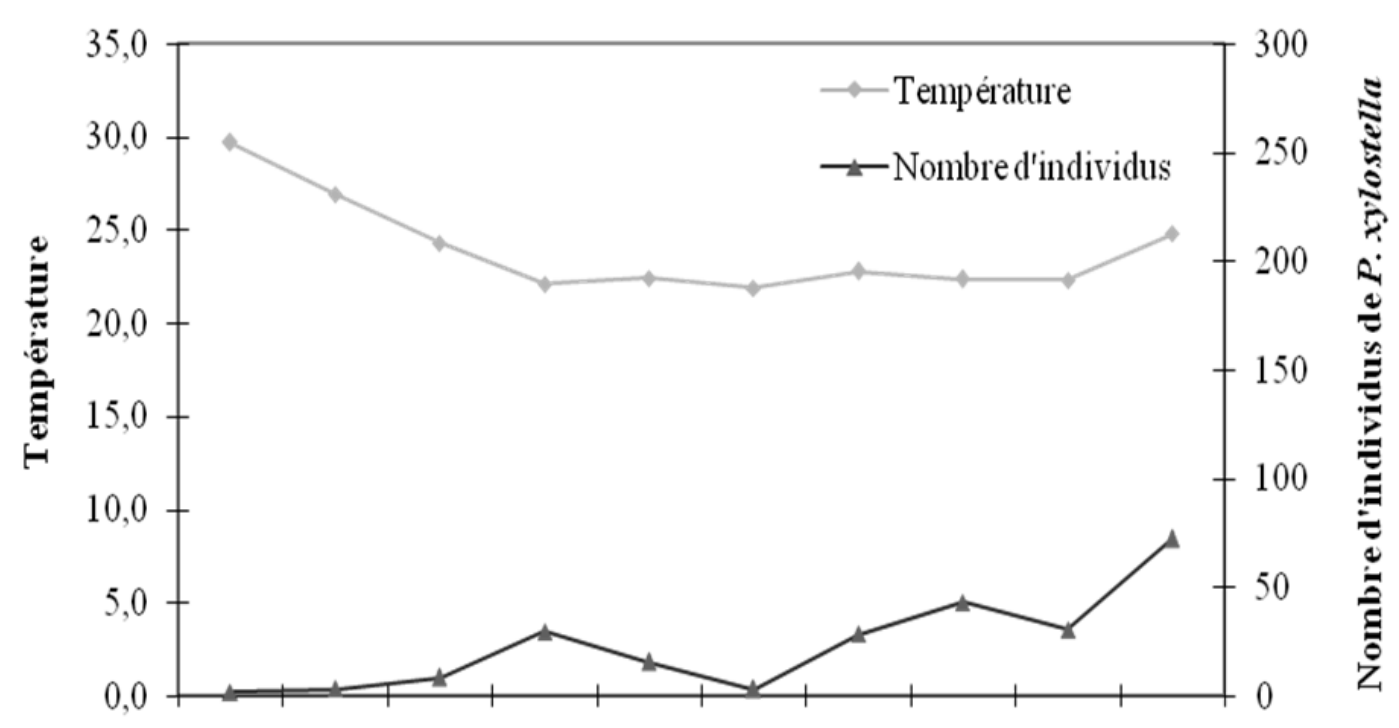

22-oct. 12-nov. 03-déc. 25-déc. 14-janv.25-févr. 18 -mars 08-avr. 29-avr. 20-mai

Date des échantillonnages

Figure 4 : Evolution du nombre de $P$. xylostella (chenilles et nymphes) suivant la température dans la zone Nord. 
Tableau 1 : Coordonnées GPS des localités des parcelles échantillonnées dans les zones Nord, Centre et Sud (Présenté sur carte Figure 1 par Qgis).

\begin{tabular}{|c|c|c|c|}
\hline \multirow[t]{2}{*}{$\mathbf{N}^{\circ}$ Parcelles } & \multirow{2}{*}{$\begin{array}{c}\text { Localités } \\
\text { d'échantillonnage } \\
\text { Zone sud }\end{array}$} & \multicolumn{2}{|c|}{ Coordonnées GPS } \\
\hline & & Latitude & longitude \\
\hline 2 & Sangalkam & 27984510 & $1,76 \mathrm{E}+08$ \\
\hline 5 & Gorom & 28717596 & $1,76 \mathrm{E}+08$ \\
\hline 7 & Der & 29029903 & $1,77 \mathrm{E}+08$ \\
\hline 10 & Mbawane & 29113474 & $1,77 \mathrm{E}+08$ \\
\hline 11 & Keur Abdou Ndoye & 28842497 & $1,77 \mathrm{E}+08$ \\
\hline 13 & Kayar & 29185628 & $1,77 \mathrm{E}+08$ \\
\hline 16 & Ndieguène & 29765029 & $1,76 \mathrm{E}+08$ \\
\hline 17 & Tieudeum & 29561621 & $1,77 \mathrm{E}+08$ \\
\hline 20 & Keur Mbir Ndao & 29759337 & $1,78 \mathrm{E}+08$ \\
\hline 21 & Lac Rose & 28186804 & $1,76 \mathrm{E}+08$ \\
\hline 102 & Tieudem & 29490364 & $1,77 \mathrm{E}+08$ \\
\hline 104 & Keur Mbir Ndao & 29750561 & $1,78 \mathrm{E}+08$ \\
\hline 106 & Ndieguene & 29788884 & $1,76 \mathrm{E}+08$ \\
\hline 108 & Ber & 29092233 & $1,77 \mathrm{E}+08$ \\
\hline 110 & Keur Abdou ndoye & 29055578 & $1,77 \mathrm{E}+08$ \\
\hline 112 & Kayar & 29187891 & $1,77 \mathrm{E}+08$ \\
\hline 114 & Gorom2 & 28744372 & $1,76 \mathrm{E}+08$ \\
\hline 116 & Lac rose & 28178523 & $1,77 \mathrm{E}+08$ \\
\hline 118 & Niaga & 27751809 & $1,76 \mathrm{E}+08$ \\
\hline \multirow[t]{2}{*}{120} & Sangalkam & 27985583 & $1,76 \mathrm{E}+08$ \\
\hline & Zone centre & & \\
\hline 24 & Santhie Ndong & 31505661 & $1,79 \mathrm{E}+08$ \\
\hline 26 & Mboro & 31637529 & $1,8 \mathrm{E}+08$ \\
\hline 27 & Touba Ndiaye & 31941293 & $1,8 \mathrm{E}+08$ \\
\hline 30 & Darou Khoudouss & 32415944 & $1,8 \mathrm{E}+08$ \\
\hline 31 & Seguel & 32246333 & $1,8 \mathrm{E}+08$ \\
\hline 33 & Ndarou Fall & 32924805 & $1,81 \mathrm{E}+08$ \\
\hline 34 & Mboro Cifop & 31845294 & $1,8 \mathrm{E}+08$ \\
\hline 35 & Diogo Ndiaye & 33052163 & $1,81 \mathrm{E}+08$ \\
\hline 37 & Darou Gueye & 32514141 & $1,81 \mathrm{E}+08$ \\
\hline 40 & Fass Boy & 32459355 & $1,81 \mathrm{E}+08$ \\
\hline 42 & Darou Ndoye & 32776239 & $1,81 \mathrm{E}+08$ \\
\hline 59 & Touba Ndiaye & 32127134 & $1,8 \mathrm{E}+08$ \\
\hline 122 & Darou Khoudouss & 32417289 & $1,8 \mathrm{E}+08$ \\
\hline 124 & Diogo Ndiaye & 33052098 & $1,81 E+08$ \\
\hline 126 & Darou Fall & 32922336 & $1,81 \mathrm{E}+08$ \\
\hline 128 & Darou Ndoye & 32796108 & $1,81 \mathrm{E}+08$ \\
\hline 130 & Fass Boy & 32462414 & $1,81 \mathrm{E}+08$ \\
\hline 132 & Darou Gueye & 32512412 & $1,81 \mathrm{E}+08$ \\
\hline 134 & seugeul & 32238422 & $1,8 \mathrm{E}+08$ \\
\hline 136 & Mboro Waou & 31921414 & $1,8 \mathrm{E}+08$ \\
\hline 138 & Mboro & 31640310 & $1,8 \mathrm{E}+08$ \\
\hline 140 & Sathie Ndong & 31666828 & $1,79 \mathrm{E}+08$ \\
\hline
\end{tabular}




\begin{tabular}{|c|c|c|c|}
\hline & Zone nord & & \\
\hline 43 & Sag & 35608080 & $1,86 \mathrm{E}+08$ \\
\hline 47 & Koura Diery & 36035957 & $1,87 \mathrm{E}+08$ \\
\hline 48 & Potou & 36167212 & $1,87 \mathrm{E}+08$ \\
\hline 50 & Gabar Diop & 36044423 & $1,87 \mathrm{E}+08$ \\
\hline 51 & Degou Niaye & 36215716 & $1,88 \mathrm{E}+08$ \\
\hline 52 & Darou mboubaye & 36396215 & $1,88 \mathrm{E}+08$ \\
\hline 54 & Mboltine & 37180290 & $1,88 \mathrm{E}+08$ \\
\hline 55 & Ndoye Dia & 37055687 & $1,88 \mathrm{E}+08$ \\
\hline 56 & Ndoye Diagne & 37252406 & $1,88 \mathrm{E}+08$ \\
\hline 58 & Kalassane & 37542188 & $1,89 \mathrm{E}+08$ \\
\hline 142 & Keur Coura Diery & 35968787 & $1,87 \mathrm{E}+08$ \\
\hline 144 & Sagal satiel & 35607515 & $1,86 \mathrm{E}+08$ \\
\hline 146 & Potou nord & 36168774 & $1,87 \mathrm{E}+08$ \\
\hline 148 & Gabar & 36078866 & $1,87 \mathrm{E}+08$ \\
\hline 152 & Mboltin & 37181908 & $1,88 \mathrm{E}+08$ \\
\hline 156 & Ndoye ndiagne & 37244749 & $1,88 \mathrm{E}+08$ \\
\hline 158 & Potou & 36119878 & $1,87 \mathrm{E}+08$ \\
\hline
\end{tabular}

Tableau 2 : Nombre d'individus (chenilles + nymphes) de P. xylostella récoltés dans les 20 parcelles de la zone sud des Niayes.

\begin{tabular}{|c|c|c|c|c|c|c|c|}
\hline $\mathbf{N}^{\circ}$ par & Cultivars & Nb ch. échan & Chen. & Ny. & Total & Moy/échan. & Moy Px/chou \\
\hline 2 & Chou & 72 & 4 & 3 & 7 & 2,33 & 0 \\
\hline 5 & Chou & 96 & 15 & 9 & 24 & 6,00 & 0 \\
\hline 7 & Tropica Cross & 96 & 47 & 25 & 72 & 18,00 & 1 \\
\hline 10 & Tropicana & 72 & 56 & 11 & 67 & 22,33 & 1 \\
\hline 11 & Tropica Cross & 72 & 113 & 89 & 202 & 67,33 & 3 \\
\hline 13 & Tropica Cross & 96 & 950 & 285 & 1235 & 308,75 & 13 \\
\hline 16 & Tropicana & 96 & 171 & 79 & 250 & 62,50 & 3 \\
\hline 17 & Tropica Cross & 120 & 110 & 50 & 160 & 32,00 & 1 \\
\hline 20 & Milor & 96 & 247 & 99 & 346 & 86,50 & 4 \\
\hline 21 & Santa & 96 & 199 & 40 & 239 & 59,75 & 2 \\
\hline 102 & Santa & 96 & 141 & 16 & 157 & 39,25 & 2 \\
\hline 104 & Santa & 96 & 236 & 7 & 243 & 60,75 & 3 \\
\hline 106 & Tropica Cross & 96 & 101 & 21 & 153 & 38,25 & 2 \\
\hline 108 & "ordinaire" & 72 & 65 & 5 & 70 & 23,33 & 1 \\
\hline 110 & Santa & 72 & 131 & 11 & 142 & 47,43 & 2 \\
\hline 112 & Santa & 96 & 99 & 74 & 173 & 43,25 & 2 \\
\hline 114 & Santa & 72 & 42 & 15 & 57 & 19,00 & 1 \\
\hline 116 & Santa & 72 & 243 & 5 & 248 & 82,67 & 3 \\
\hline 118 & Santa & 96 & 137 & 23 & 160 & 40,00 & 2 \\
\hline 120 & Chou & 72 & 108 & 18 & 126 & 42,00 & 2 \\
\hline
\end{tabular}


Tableau 3 : Nombre d'individus (chenilles + nymphes) de $P$. xylostella récoltés dans les 22 parcelles de la zone centre des Niayes.

\begin{tabular}{lccccccc}
\hline $\mathbf{N}^{\circ}$ par & Cultivar & Nb ch. échan & Chen. & Ny. & Total & Moy/échan. & Moy Px/chou \\
\hline 24 & Tropicana & 72 & 270 & 88 & 358 & 119,33 & 5 \\
26 & Tropicana & 96 & 6 & 3 & 9 & 2,25 & 0 \\
27 & Tropica Cross & 96 & 1 & 0 & 1 & 0,25 & 0 \\
30 & Tropicana & 96 & 4 & 4 & 8 & 2,00 & 0 \\
31 & Tropicana & 96 & 131 & 52 & 183 & 45,75 & 2 \\
33 & Tropicana & 96 & 18 & 5 & 23 & 5,75 & 0 \\
34 & Tropica Cross & 96 & 63 & 12 & 75 & 18,75 & 1 \\
35 & Tropicana & 72 & 43 & 6 & 49 & 16,33 & 1 \\
37 & Tropicana & 96 & 29 & 8 & 37 & 9,25 & 0 \\
40 & Tropica Leader & 96 & 15 & 8 & 23 & 5,75 & 0 \\
42 & Tropicana & 96 & 9 & 5 & 14 & 3,60 & 0 \\
59 & Tropicana & 96 & 210 & 123 & 333 & 83,25 & 3 \\
122 & Tropicana & 72 & 335 & 42 & 377 & 125,00 & 5 \\
124 & Santa & 96 & 88 & 17 & 105 & 26,25 & 1 \\
126 & Dragon & 96 & 90 & 24 & 138 & 34,50 & 1 \\
128 & Santa & 96 & 129 & 9 & 155 & 38,75 & 2 \\
130 & Santa & 72 & 72 & 13 & 85 & 28,33 & 1 \\
132 & Santa & 72 & 29 & 12 & 61 & 20,33 & 1 \\
134 & Tropicana & 96 & 70 & 25 & 107 & 26,75 & 1 \\
136 & Tropicana & 72 & 18 & 8 & 26 & 8,67 & 0 \\
138 & Santa & 96 & 138 & 12 & 157 & 39,25 & 2 \\
140 & Tropicana & 72 & 36 & 10 & 46 & 15,33 & 1 \\
\hline $\mathbf{N}^{\circ}$ par : Numéro de la parcelle $;$ Chen. : Chenilles ; Ny. : Nymphes ; Moy/échan : Moyenne de $P$. & \\
$x y l o s t e l l a /$ échantillonnage ; Moy Px/chou : Moyenne de P. xylostella/chou ; Nb ch. échan: Nombre de choux échantillonnés
\end{tabular}

Tableau 4 : Nombre d'individus (chenilles + nymphes) de P. xylostella récoltés dans les 17 parcelles de la zone nord des Niayes.

\begin{tabular}{lccccccc}
\hline $\mathbf{N}^{\circ}$ par & Cultivar & Nb ch. échan & Chen. & Ny. & Total & Moy/échan. & Moy Px/chou \\
\hline 43 & Tropica Cross & 96 & 103 & 48 & 151 & 37,75 & 2 \\
47 & Tropicana & 96 & 11 & 2 & 13 & 3,25 & 0 \\
48 & Taizé & 72 & 19 & 4 & 23 & 7,67 & 0 \\
50 & Tropica Cross & 96 & 15 & 9 & 24 & 6,00 & 0 \\
51 & Fabula & 96 & 82 & 18 & 100 & 25,00 & 1 \\
52 & K K Cross & 96 & 14 & 1 & 15 & 3,75 & 0 \\
54 & Tropica Cross & 96 & 4 & 3 & 7 & 1,75 & 0 \\
55 & Tropica Cross & 96 & 1 & 0 & 1 & 0,25 & 0 \\
56 & "Ordinaire" & 96 & 35 & 51 & 86 & 21,50 & 1 \\
58 & Tropica Cross & 96 & 12 & 17 & 29 & 7,25 & 0 \\
142 & "Ordinaire" & 72 & 118 & 48 & 166 & 55,33 & 2 \\
144 & Tropica Cross & 96 & 23 & 6 & 47 & 11,75 & 0 \\
146 & Tropicana & 96 & 69 & 14 & 83 & 20,75 & 1 \\
148 & Taizé & 72 & 194 & 14 & 208 & 69,33 & 3 \\
152 & Tropica Cross & 72 & 46 & 28 & 90 & 30,00 & 1 \\
156 & Taizé & 48 & 9 & 10 & 83 & 41,50 & 2 \\
158 & Taizé & 72 & 114 & 23 & 137 & 45,67 & 2 \\
\hline$N^{\circ}$ par : Numéro de la parcelle ; Chen. : Chenilles ; Ny. : Nymphes ; Moy/échan : Moyenne de $P$. xylostella/échantillonnage \\
Moy Px/chou : Moyenne de $P$. xylostella/chou; Nb ch. échan: Nombre de choux échantillonnés
\end{tabular}


Tableau 5 : Sensibilité des différents cultivars de chou utilisés par les agriculteurs des Niayes.

\begin{tabular}{lccc}
\hline Zones & Cultivars & Nombre chou/échantillon. & Nombre individu/chou \\
\hline Sud & Tropica Cross & 480 & 3,80 \\
& Tropicana & 168 & 1,89 \\
& Santa & 696 & 2,04 \\
& Milor & 96 & 3,60 \\
\hline Centre & Tropica Cross & 196 & 0,38 \\
& Tropicana & 1128 & 1,32 \\
& Tropica Leader & 96 & 0,24 \\
& Santa & 456 & 1,34 \\
& Dragon & 96 & 1,44 \\
\hline Nord & Tropica Cross & 648 & 0,54 \\
& Tropicana & 192 & 0,50 \\
& K K Cross & 96 & 0,16 \\
& Taizé & 264 & 1,71 \\
& Fabula & 96 & 1,04 \\
\hline
\end{tabular}

\section{DISCUSSION}

D'après les résultats obtenus, la zone sud est la plus infestée par P. xylostella parmi les trois zones étudiées. Cette tendance peut être expliquée par le fait qu'elle présente une zone horticole beaucoup plus intensive et des parcelles beaucoup plus rapprochées, mais également par le fait que le chou est cultivé dans cette zone pendant toute l'année, contrairement à la zone nord où elle est entrecoupée par la culture de l'oignon. Selon Asare-Bediako et al. (2010) ce dernier a un effet répulsif contre les adultes de la Teigne et réduit les populations de chenilles de $P$. xylostella quand il est associé avec du chou. D'après Sarker et al. (2007), l'ail et l'oignon cultivées en intercalaire avec le chou réduit considérablement la population de pucerons, il pourrait en être de même dans le cas des chenilles de $P$. xylostella. De même, Saïd and Itulya (2003) ont indiqué que l'odeur de l'oignon est capable de repousser les adultes de $P$. xylostella lors de leur installation sur le chou. En effet, l'ail et l'oignon produisent un composé alliacé, l'allyle epropyl-disulfure, qui est responsable de la répulsion des ravageurs. D'autre part, il semblerait que la culture intercalaire permet également de réduire l'attaque des ravageurs parce que l'effet de la culture non hôte, agit comme une barrière physique à la circulation des insectes ravageurs (Sheehan, 1986).

Dans la zone nord, on constate un grand nombre de surfaces en friches entrecoupant les parcelles de choux. Celles-ci pourraient avoir comme effet l'isolement des parcelles cultivées qui pourraient perturber l'installation des adultes du ravageur en provoquant comme précédemment une confusion olfactive (Sarker et al., 2007). On pourrait en déduire qu'une zone où les parcelles sont distantes et séparées par des friches, plus ou moins étendues ne seraient pas forcément favorable à $P$. xylostella.

L'espèce $P$. xylostella migre de façon passive à la faveur des vents (Honda, 1992 ; Honda et al., 1992). Plusieurs auteurs montrent que ces migrations sont incontrôlables quant à leur destination finale (Zalucki and Furlong, 2011; Wei et al., 2013), ce qui entraîne une infestation des nouvelles parcelles d'une façon aléatoire et imprévisible.

Au centre, comme dans la zone nord, la présence de friches peut également être préjudiciable à l'implantation des populations de la Teigne. En général, les ravageurs herbivores en présence de plantes non hôtes sont perturbés pour la recherche de la plante 
hôte et finissent par quitter la zone (Kareiva, 1985; Bernays, 1999). Un grand nombre de parcelles de cette zone sont cultivées sur les dunes orientées du côté de la mer. La présence de vents souvent forts et surtout réguliers provenant de celle-ci, peuvent freiner les populations d'adultes portées par ceux venant de la terre (Chapman et al., 2002 ; Zalucki et Furlong, 2011).

Dans les trois zones, les attaques sont très hétérogènes au niveau de l'importance des infestations avec une moyenne d'individus (chenilles et nymphes) récoltés variant de zéro à 13 par chou pendant la durée de la culture, ce qui n'a pas eu d'impact réel sur la production pour les agriculteurs. Cependant, certains auteurs considèrent que la présence d'une chenille L4 par chou suffit à altérer et rendre invendable la pomme (Shelton et al., 1983; Maltais et al., 1998). Il faut noter que les normes de vente du marché locale sénégalais ne sont pas si drastiques que cela.

La variation des infestations observée entre les parcelles, peut être liée à l'utilisation des pesticides. Certaines parcelles sont traitées par une formulation biologique à base de Bacillus thuringiensis Berliner, mais également avec un produit naturel à base de graines de " l'arbre de Neem" (m.a. Azadirachtine). Ces produits ont montré une réelle efficacité contre les chenilles de la Teigne dans nos conditions (Sow, 2013), mais également ailleurs, comme au Bénin (Goudegnon et al., 2000), et au Kenya (Löhr and Kfir, 2004). D'autres subissent des applications assez régulières de produits inefficaces et interdits comme le diméthoate et l'ométhoate, le métamidophos, des herbicides. Ces pratiques favorisent malheureusement la résistance aux insecticides ( $\mathrm{Li}$ et al., 2012). Enfin, on ne peut juger l'efficacité d'autres pesticides tels que le "Tersen", le "Bomec", le "Terpride", le "Conquest", le "Pashmine", le "Calfos", dont nous ignorons la matière active. Il est probable que ces genres de produits détruisent les ennemis naturels et contaminent l'environnement (Furlong et al., 2012).
Il se pourrait que les haies aussi jouent un rôle de barrière pour les adultes, ou limite la distance de migration des ravageurs (Kührt et al., 2006 ; Ricci et al., 2009). Mais dans notre cas, du fait du faible degré d'infestation, leur impact n'est pratiquement pas décelable.

Le type d'arrosage employé par les agriculteurs peut influencer la dynamique des chenilles du ravageur. En effet, certains auteurs (Tabashnik et al., 1986 ; Sow, 2013) mentionnent que les fortes pluies réduisent les populations de chenilles de la Teigne. Certains auteurs ont montré que les fortes pluies sont un facteur important de mortalité du fait du lavage des œufs, des larves et des nymphes sur les feuilles (Baidoo et al., 2012). Ce phénomène a été étudié et l'on observe qu'une heure de forte pluie, détruit $95,3 \%$ des larves de stade $1,72 \%$ de stade $2,60,7 \%$ de stade 3 et $42,7 \%$ de stade 4 de $P$. xylostella (Koboriand Amano, 2003). L'arrosage par aspersion des cultures (surtout dans la zone centre) à l'aide d'une lance à eau, pourrait produire le même effet, car ce type d'arrosage réduit significativement les infestations de $P$. xylostella de 40 à $60 \%$ en une année d'irrigation aux Etats-Unis (McHughand Foster, 1995). Par ailleurs, (Baidoo et al., 2012) ont affirmé que les faibles précipitations favorisent l'augmentation du nombre de P. xylostella.

En général, les populations de la Teigne causent beaucoup plus de dégâts dans les zones tropicales que tempérées (Ansari et al., 2010 ; Guo et al., 2010). Au Sénégal, les populations de $P$. xylostella augmentent lors de la première saison où la température varie entre 20 et $25{ }^{\circ} \mathrm{C}$ et semblent diminuer dès que celle-ci remonte à plus de $30^{\circ} \mathrm{C}$ (Sow, 2013). Lors de notre étude, une seule corrélation a été notée à la deuxième saison nord entre les fluctuations de température et le nombre de chenilles. Ce fait montre que les infestations varient aléatoirement selon la saison. Cela est probablement dû au faible nombre de chenilles récoltées lors de nos échantillonnages durant les deux saisons. 
Nous signalerons quand même que Guo and Qin, 2010, au cours d'études effectuées en Chine, n'ont obtenu aucune corrélation entre la température et l'importance des populations de $P$. xylostella. Ces résultats témoignent de l'importance de la fluctuation de la température due aux changements climatiques au niveau mondial ces dernières années (Loko et al., 2013 ; Gbaguidi et al., 2015).

Les glucosinolates sont des composés soufrés produits par les choux qui attirent les femelles pour pondre et sont appétant envers les jeunes chenilles (Hamilton et al., 2005 ; Haugen et al., 2008 ; Furlong et al., 2012). Mewis et al. (2002) a montré que certains cultivars de chou produisent plus de glucosinolates que d'autres, ce qui les rend plus sensibles aux attaques des ravageurs. Actuellement, il existe des variétés de choux moins sensibles aux attaques des chenilles de $P$. xylostella car ils produisent des toxines responsables de mécanisme d'antibiose (Eigenbrode and Shelton, 1992), ou par des changements de la structure des cires épicuticulaires qui diminuent l'appétence des feuilles (Shimabuku et al., 1997). Dans notre cas, Tropica Cross, Tropica Leader et KK Cross sont issus du même pool génétique et sélectionnés pour la zone tropicale d'Afrique de l'Ouest et Centrale. Ils semblent avoir une bonne tolérance quand les attaques de la Teigne sont faibles, comme c'est le cas dans la zone centre et nord. Par contre, sa tolérance diminue quand les attaques augmentent, ce qui se vérifie dans la zone sud. Le cultivar Tropicana, cultivé en Martinique et sélectionné pour un meilleur développement en période fraîche, semble bien se comporter à cette époque où la température est inférieure à $30{ }^{\circ} \mathrm{C}$, mais comme les cultivars précédents, sa tolérance diminue quand la pression du ravageur augmente.

Devant le comportement imprévisible des infestations de ce ravageur au niveau des parcelles cultivées (Zalucki and Furlong, 2011; Wei et al., 2013), la lutte doit être adaptée pour maintenir les populations de chenilles à un seuil économique acceptable pour le producteur. On connaît les risques pour la santé humaine et l'environnement, découlant des applications anarchiques d'insecticides (Furlong et al., 2012), utilisés par des acteurs souvent mal informés voire ignorant des effets néfastes de ces pesticides et nous savons également que la réussite des programmes de libérations d'auxiliaires biologiques est très coûteuse et nécessite une compétente technique importante qui est malheureusement absente chez ces producteurs. La solution à ces problèmes serait en premier lieu de favoriser la lutte biologique par conservation de la faune utile en limitant les applications insecticides à l'aide de formulations à base de produits naturels (huile de "neem") ou biologiques (B.thuringiensis), mais également effectuer des recherches sur les effets que pourraient apporter les plantes compagnes à travers des espèces maraîchères cultivées qui possèdent un effet piège (Moutarde chinoise, Pak choi), répulsif (oignon, ail, basilic), ou neutre (salade, carotte...), mais qui pourraient perturber les femelles de $P$. xylostella à la recherche de sites de ponte (Sheehan, 1986). Ces espèces cultivées pourraient également apporter un supplément de revenu aux producteurs. La mise en place de cette stratégie serait également en phase avec les compétences horticoles que possède le producteur de spéculations légumières.

\section{REMERCIEMENTS}

Nous tenons à remercier A. Dabo, A. N. Fall, E. Tendeng et A. Dia (étudiants de master), et FPMN (Fédération des Producteurs Maraîchers de la zone des Niayes) de l'aide pour l'enquête, suivi sur le terrain et la collecte des insectes.

\section{REFERENCES}

Ansari MS, Ahmad T, Ali H. 2010. Effect of Indian mustard on feeding, larval survival and development of Plutella xylostella at constant temperatures. Entomol. Res., 40: 
182-188.

DOI: $10.1111 / \mathrm{j} .1748$ 5967.2010.00274.x

Asare-Bediako E, Addo-Quaye AA, Mohammed A. 2010. Control of diamondback moth (Plutella xylostella) on cabbage (Brassicaoleracea var capitata) using intercropping with nonhost crops. Am. J. Food Technol., 5(4): 269-274. DOI: http://dx.doi.org/ 10.3923/ajft.2010.269.274

Badiane D, Gueye MT, Coly EV, Faye O. 2015. Gestion intégrée des principaux ravageurs du cotonnier au Sénégal et en Afrique occidentale. Int. J. Biol. Chem. Sci., 9(5): 2654-2667. DOI : http://dx.doi.org/10.4314/ijbcs.v9i5.36

Baidoo PK, Mochiah MB, Apusiga K. 2012. Onion as a Pest Control Intercrop in Organic Cabbage (Brassica oleracea) Production System in Ghana. Sustainable Agriculture Research, 1(1): 36. DOI:http://dx.doi.org/10.5539/sar.v1n1p3 6

Bernays EA. 1999. When host choice is a problem for a generalist herbivore, experiments with the whitefly. Bemisia tabaci. Ecol. Entomol., 24: 260-267. DOI: 10.1046/j.1365-2311.1999.00193.x

Cai HJ, Li S, Ryall K, You M, Lin S. 2011. Effects of intercropping of garlic or lettuce with Chinese cabbage on the development of larvae and pupae of diamondback moth (Plutella xylostella). Afr. J. Agri. Res., 6(15): 3609-3615. DOI: 10.5897/AJAR11.789

Chapman JW, Reynolds DR, Smith AD, Riley JR, Pedgley DE, Woiwod IP. 2002. Highaltitude migration of the diamondback moth Plutella xylostella to the UK: a study using radar, aerial netting, and ground trapping. Ecol. Entomol., 27: 641-50. DOI: 10.1046/j.13652311.2002.00472.x

Chilcutt CF, Tabashnik BE. 1997. Hostmediated competition between the pathogen Bacillus thuringiensis and the parasitoid Cotesia plutellae of the diamondback moth (Lep: Plutellidae). Environ. Entomol., 26: 38-45. DOI: 10.1093/ee/26.1.38

Eigenbrode SD, Shelton AM. 1992. Resistance to diamondback moth in Brassica: Mechanisms and potential for resistant cultivars. In Diamondback moth and other crucifers pests, Talekar NS (ed). Proceedings of the second International Workshop, Tainan, Taiwan, 10-14 December 1990, Asian Vegetable Research and Development Center. p. 213-224.

FAOSTAT. 2012. Production statistics. Rome: FAO. http://faostat.fao.org/ site/567/

FAO. 2015. FAOSTAT database. Online at:http://faostat3.fao.org/home/E [accessed 23.06.15].

Furlong MJ, Wright J, Dosdall LM. 2012. Diamondback Moth Ecology and Management: Problems, Progress and Prospects. Annu. Rev. Entomol., 58: 51741. DOI: 10.1146/annurev-ento-120811153605

Gbaguidi AA, Faouziath S, Orobiyi A, Dansi M, Akouegninou BA, Dansi A. 2015. Connaissances endogènes et perceptions paysannes de l'impact des changements climatiques sur la production et la diversité du niébé (Vigna unguiculata (L.)Walp.) et $\mathrm{du}$ voandzou (Vigna subterranea (L) Verdc.) au Bénin. Int. J. Biol. Chem. Sci., 9(5): 2520-2541. DOI : http://dx.doi.org/10.4314/ijbcs.v9i5.23

Goudegnon AE, Kirk AA, Schiffers B, Bordat D. 2000. Comparative effects of deltamethrin and neem kernel solution treatments on diamondback moth and Cotesia plutellae (Hym. Braconidae) parasitoid populations in the Cotonou peri-urban area in Benin. J. Appl. Entomol, $\quad$ 124: 141144.DOI: 10.1046/j.1439-0418.2000. 00461.x

Guo S, Qin Y. 2010. Effects of temperature and humidity on emergence dynamics of 
Plutella xylostella (Lepidoptera; Plutellidae). J. Econ. Entomol., 103(6): 2028-2033. DOI: http://dx.doi.org/10. 1603/EC09251

Grzywacz D, Rossbach A, Rauf A, Russell DA, Srinivasan R, Shelton AM. 2010. Current control methods for diamondback moth and other brassica insect pests and the prospects for improved management with lepidopteran-resistant $B t$ vegetable brassicas in Asia and Africa. Crop Prot., 29: 68-79. DOI: 10.1016/j.cropro. 2009.08.009

Hamilton AJ, Endersby NM, Ridland PM, Zhang J, Neal M. 2005. Effects of cultivar on oviposition preference, larval feeding and development time of diamondback moth, Plutella xylostella (L.) (Lepidoptera: Plutellidae), on some Brassica oleracea vegetables in Victoria. Austr. J. Entomol., 44: 284287.DOI: $10.1111 / \mathrm{j} .1440-6055.2005$. 00468.x

Haugen R, Steffes L, Wolf J, Brown P. Matzner S, Siemens DH. 2008. Evolution of drought tolerance and defense: dependence of tradeoffs on mechanism, environment and defense switching. Oikos, 117: 231-244. DOI: 10.1111/j.2007.0030-1299.16111.x

Hill TA, Foster RE. 2000. Effect of insecticides on the diamondback moth (Lepidoptera: Plutellidae) and its parasitoid Diadegma insulare (Hymenoptera: Ichneumonidae). J. Econ. Entomol., 93(3): 763-768. DOI: http://dx.doi.org/10.1603/0022-049393.3.763

Hooks CRR, Johnson MW. 2003. Impact of agricultural diversification on the insect community of cruciferous crops. Crop Prot., 22: 223-238. DOI: 10.1016/S02612194(02)00172-2

Honda KI. 1992. Hibernation and migration of diamondback moth in Northern Japan. In: Talekar N. S. (Ed.) Diamondback moth and other crucifer pests: Proceedings of the second International Workshop, Tainan, Taiwan, 10-14 December 1990, AVRDC, 43-50.

Honda KI, Miyahara Y, Kegasawa K.1992. Seasonal abundance and the possibility of spring immigration of the diamondback moth, Plutella xylostella (L.) (Lep: Plutellidae), in Morioka City, Northern Japan. Appl. Entomol. Zool., 27: 517-524. DOI: http://doi.org/10.1303/aez.27.517

Huang Z, Ali S, Ren SX, Wu JH. 2010. Effect of Isaria fumosoroseus on mortality and fecundity of Bemisia tabaci and Plutella xylostella. Insect Sci. Appl., 17: 140-148. DOI: 10.1111/j.1744-7917.2009.01299.x

Kareiva PM. 1985. Finding and losing host plants by Phyllotreta, patch size and surrounding habitat. Ecol., 66: 18091818. DOI: $10.2307 / 2937376$

Kobori Y, Amano H. 2003. Effect of rainfall on a population of diamondback moth Plutella xylostella (Lepidoptera: Plutellidae). Jap. Appl. Entomol. Zool., 38(2): 249-253. DOI: http://doi.org/ 10.1303/aez.2003.249

Kührt U, Samietz J, Höhn H, Dorn S. 2006. Modelling the phenology of codling moth: influence of habitat and thermoregulation. Agri. Ecosyst. Environ., 117: 29-38.DOI:10.1016/ j.agee.2006.03.003

Li ZY, Zalucki MP, Bao HL, Chen HY, Hu ZD, Feng X. 2012. Population dynamics and 'outbreaks' of diamondback moth, Plutella xylostella, in Guangdong Province, China: climate or the failure of management? J. Econ. Entomol., 105: 739-52. DOI: http://dx.doi.org/10.1603/ EC11384

Loko YL, Dansi A, Agre AP, Akpa N, Dossou-Aminon1 I, Assogba P, Dansi M, Akpagana K, Sanni A. 2013. Perceptions paysannes et impacts des changements climatiques sur la production et la diversité variétale de l'igname dans la zone aride du Nord-Ouest du Bénin. Int. 
J. Biol. Chem. Sci., 7(2): 672-695. DOI: http://dx.doi.org/10.4314/ijbcs.v7i2.23

Löhr B, Kfir R. 2004. Diamondback moth Plutella xylostella in Africa: a review with emphasis on biological control. In Improving Biocontrol of Plutella xylostella, Bordat D, Kirk AA (eds). CIRAD, Proceedings of the International Symposium in Montpellier, France, 2124 Oct 2002, pp. 71-84.

Macharia I, Löhr B, De Groote H. 2005. Assessing the potential impact of biological control of Plutella xylostella (diamondback moth) in cabbage production in Kenya. Crop Prot., 24 : 981-989.DOI:10.1016/j.cropro.2005. 02.005

McHugh JJ, Foster RE. 1995. Reduction of diamondback moth (Lepidoptera: Plutellidae) infestation in head cabbage by overhead irrigation. J. Econ. Entomol., 88: $162-168 . \quad$ DOI: http://dx.doi.org/10.1093/jee/88.1.162

Maltais PM, Nuckle JR, Leblanc PV. 1998. Economic threshold for three lepidopterous larval pests of fresh-market cabbage in South easthern New Brunswick. J. Econ. Entomol., 91: 699707.DOI: http://dx.doi.org/10.1093/jee/ 91.3.699

Mewis I, Ulrichs C, Schnitzler WH. 2002. The role of glucosinates and their hydrolysis

products in oviposition and host plants finding by Cabbage webworm, Hellula undalis. Entomol. Exp. Appl., 105: 129-139. DOI: 10.1046/j.1570-7458.2002.01041.x

Ricci B, Franck P, Toubon JF, Bouvier JC, Sauphanor B, Lavigne C. 2009. The influence of landscape on insect pest dynamics: a case study in southeastern France. Landscape Ecol., 24: 337-349. DOI:10.1007/s10980-008-9308-6

Said M, Itulya FM. 2003. Intercropping and nitrogen management effects of diamondback moth and yields of collards in the highlands of Kenya. Afr. Crop. Sci. J., 2: $\quad 35-42$. $\quad$ DOI: http://www.bioline.org.br/abstract?id=cs0 3005

Sarker PK, Rahman MM, Das BC. 2007. Effect of intercropping of mustard with onion and garlic on aphid population and yield. J. Biol. Sci., 15: 35-40. DOI: 10.3329/jbs.v15i0.2200

Sarfraz M, Dosdall LM, Keddie BA. 2005. Spinosad: a promising tool for integrated pest management. Outlooks Pest Manage, 16: 78-84. DOI:http://dx.doi.org/10.1564/ 16 apl09

Sarfraz M, Keddie BA. 2005. Conserving the efficacy of insecticides against Plutella xylostella (L.) (Lep. Plutellidae). J. Appl. Entomol., 129: 149-157. DOI: 10.1111/j.1439-0418.2005.00930.x

Sheehan W. 1986. Response of specialist and naturalist natural enemies to agroecosystem diversification. A selective review. Environ. Entomol., 15: 456-461. DOI: http://dx.doi.org/10.1093/ ee/15.3.456

Shelton AM, Sears MK, Wyman JA, Quick TC. 1983. Comparaison of action thresholds for lepidopterous larvae on fresh-market cabbage. J. Econ. Entomol., 76: $196-199 . \quad$ DOI: http://dx.doi.org/10.1093/jee/76.1.196

Shelton AM. 2004. Management of the Diamondback Moth: All Over Again? The Management of Diamondback Moth and Other Crucifer Pests, Endersby N, Ridland PM (eds). Melbourne, Australia 26-29 November 2001; 3-8.

Shelton AM, Roush RT, Wang P, Zhao JZ. 2007. Resistance to insect pathogens and strategies to manage resistance: An update. In Field Manual of Techniques in Invertebrate Pathology. Kluwer Academic Press: Kluwer; 793-811.

Shimabuku RS, Mau RFL, Gusumuka-minuto L. 1997. Diamondback moth: feeding preference among commercial varieties of head cabbage. In The Management of Diamondback moth and other crucifer pests, Sivapragasam A, Loke WH, 
Hussan AK, Lim GS (eds). Proceedings of the third International Workshop, Kuala Lumpur, Malaysia, 29 October 1 November 1996, Malaysian Agricultural Research and Development Institut (MARDI) \& Malaysian Plant Protection Society, p. 295-297.

Sow G, Diarra K, Arvanitakis L, Bordat D. 2013. Relationships between the diamondback moth, climatic factors, cabbage crops and natural enemies in a tropical area. Folia. Hort., 25(1): 312.DOI: 10.2478/fhort-2013-0001

Tabashnik BF, Mau RFL. 1986. Suppression of Diamondback moth (Lepidoptera: Plutellidae) oviposition by overhead irrigation. J. Econ. Entomol., 79: 189191. DOI: http://dx.doi.org/10.1093/jee/ 79.1.189

Talekar NS, Shelton AM. 1993. Biology, Ecology and management of the diamondback moth. Ann. Rev. Entomol.,
38: 275-301. DOI: 10.1146/annurev. en.38.010193.001423

Warwick SI, Francis A, Mulligan GA. 2003. Brassicaceae of Canada. Government of Canada. Available: http://www.cbif. gc.ca/spp_pages/brass/index_e.php.

Wei SJ, Shi BC, Gong YJ, Jin GH, Chen XX. 2013. Genetic Structure and Demographic History Reveal Migration of the Diamondback Moth Plutella xylostella (Lepidoptera: Plutellidae) from the Southern to Northern Regions of China. PLoS ONE., 8(4): 59-65. DOI:10.1371/journal.pone.0059654

Zalucki JM, Furlong MJ. 2011. Predicting outbreaks of a major migratory pest: an analysis of diamondback moth distribution and abundance revisited. Annu. Rev. Entomol., 58: 8-14. 